pact object (black hole) and the accretion rate onto it.

In the final session, Scheuer led a discussion of some of the central questions. Is there bulk relativistic motion? If so, how much more complicated than the simple relativistic jet must it be to account for all the facts? What bends jets, are they intrinsically one-sided and how fast are the arc second jets? What is the relationship between VLBI jets and large-scale structures? What are the family relationships?
It seems that 12 years of intensive study of superluminal motion have raised more questions than they have answered. Perhaps the next 12 years, with the prospect of a dedicated VLBI array studying the structural changes in large numbers of objects, will bring answers to some of these questions.

Richard Porcas is at the Max-Planck-Institut für Radioastronomie, Auf dem Hügel 69, D-5300, Bonn 1.

\title{
Schistosomiasis
}

\section{Plants that kill snails and prospects for disease control}

\section{from G. Webbe and J.D.H. Lambert}

MOLLUSCICIDES play an important part in the effective control of schistosomiasis. Partly as a consequence of the rising costs of synthetic molluscicides, there has been an increasing interest in plants showing molluscicidal properties. The plants Phytolacca dodecandra, Ambrosia maritima and Anacardium occidentalis are among several which have shown promising molluscicidal activity. At present certain saponins, specifically those with an oleonic acid derivative, seem to offer the greatest potential as molluscicides.

A significant reduction of schistosomal disease may be possible with the use of the new efficient drugs. Although chemotherapy will undoubtedly play a vital part in reducing the severity of disease and contamination in the environment, as well as helping to control the transmission, the effect of population-based chemotherapy as the sole method of controlling transmission is unknown. It is however considered that, in many areas, a combination of snail control and chemotherapy will be the quickest and the most cost-effective way to reduce the prevalence and intensity of infection and to control transmission.

Even so, where schistosomiasis is not a clear priority, the high cost of drugs, molluscicides and other methods will clearly make it very difficult for poorer countries, even in major endemic areas, to establish control programmes within the constraints of small health budgets. Control programmes will not be seriously undertaken, nor will substantial progress be achieved in such areas, unless overall costs are generally less than $\$ 1.0$ per caput of the population protected. It is therefore important to maintain efforts to develop lower-cost technology including drugs, molluscicides and their application.

The impetus to develop plant molluscicides is largely due to a combination of the high cost of imported synthetic compounds and increasing concern about the possible development of resistance to them, and their toxicity to non-target organisms. Plant molluscicides will probably be most useful in areas where transmission is predominantly focal, rather than widespread through large areas in which synthetic molluscicides will continue to be used. It is believed that plant molluscicides could play an important part in integrated programmes at the village level and in selfhelp schemes.

Since the 1930 s, more than 1,000 plant species have been tested for molluscicidal activity, including nearly 600 in China, where a water extract of the oil of tea-cake (Thea oleosa) has been used as a molluscicide and plant fertilizer, and the powdered residue as a cercariacide in the footwear of farmers. The most thoroughly studied plant molluscicide is one derived from the berries of $\boldsymbol{P}$. dodecandra (endod), an aqueous extract of which has been used for snail control in Ethiopia. In the Sudan, a water extract of the seed of Croton macrostachys has high molluscicidal activity, as has that of Jatropha curcas, while in Egypt trials are in progress using $A$. maritima. The crushed shells of the cashew nut ( $A$. occidentalis) are being used for snail control in Mozambique.

Ten other species of Ambrosia, which is essentially an American genus, contain ambrosia and/or damsin, the two sesquiterpene lactones isolated from $A$. maritima. Some 42 molluscicidal compounds have been isolated from plants, and in addition to saponins, thought until recently to be the major active molluscicidal ingredients, flavenols, alkaloids, terpenoids and other groups have produced encouraging results. The recent finding that bidesmotic saponins and some dammarane clycosides and alkaloid saponins are inactive may help to explain the variation in the results obtained by different investigators. Further studies of other structure-activity relationships of compounds may result in the identification of plants with more selective effects. Molluscicidal compounds, like other phytotoxins, may be present and synthesized more frequently in some plant groups than in others: thus, sesquiterpene lactones are most common in the Compositae, although triterpernoid saponins are widespread in nature.

The relatively high proportion (58 per cent) of active species among the 491 species belonging to 105 families recently tested is apparently due to the selection of medicinal plants for primary screening by most investigators. The highest proportion of molluscicidal species is in the Families Phytolaccacae followed by the Mimosaceae, Polygonaceae, Verbenaceae and Caesalpinaceae. A large proportion of Euphorbiaceae are also highly active.

There is little information on the distribution of the molluscicidal principles in different plant parts, which might have predictive value, owing to the failure of most investigators to study whole plants systematically. In the Phytolaccaceae species, the berries are most active, the leaves less so and the roots not at all, which agrees with the distribution of saponins and inactive alkaloids. In most species in the Family Euphorbiaceae, the highest activity is in the seeds, and in the Solanaceae, in the leaves and fruits. Thus while efforts should be made to test all parts of the plant for molluscicidal activity, clearly for sustained use in snail control programmes it will be best in the case of perennial plants to concentrate on regenerating leaves, fruits and flowers rather than on roots, stems and non-regenerating bark.

Little is known about snail metabolism or physiology which can be used to predict the structure of potential synthetic molluscicides, let alone substances of plant origin. The known active principles of plant molluscicides are water-soluble and act on the snail surface membrane, the dose-response of saponins being an all-ornone phenomenon over a very narrow range. In considering the biochemistry of recognized molluscicidal compounds of plant origin, saponins have been emphasized, but others should be considered including tannins, flavenoids, alkaloids and terpenoids.

The availability and accessibility of information on the geographical distribution and ecosystem of molluscicidal plants in America and Europe will be extremely valuable for future research. The use of toxic plants for agricultural as well as vector control purposes may be possible; and the frequent absence of aquatic snails in the presence of specific plants may suggest which plants may be suitable for biological control.

The cultivation of plants on a large scale exclusively for snail control has not yet been attempted. The agronomic success achieved in the 1970 s with $P$. dodecandra would suggest that, at least in Ethiopia, cultivation at the village level is feasible. $\square$

G. Webbe and J.D.H. Lambert are in the UNDP/World Bank/WHO Special Programme for Research and Training in Tropical Diseases at the London School of Hygiene and Tropical Medicine, Winches Farm Field Station, St Albans, Herts. 